\title{
A Trust Valuation Model Based on Game Theory in Social Network
}

\author{
Xianqin $\mathrm{Li}^{1}$, a , Suming $\mathrm{Li}^{1, \mathrm{~b}}$ \\ ${ }^{1}$ School of National Education, Nanchang Institute of science \& Technology, Nanchang, 330108, \\ China; \\ alunwenzju@163.com, ${ }^{\text {b }}$ iefengzju@163.com
}

Keywords: Game Theory, Feedback Mechanisms, Authority Value, Trust Valuation.

\begin{abstract}
In order to solve malicious evaluation problems in evaluation model, this paper proposes an improved evaluation model with feed incentive based on game theory. The model introduces user's authority into the recommendation system based on content, which can adjust feedback function dynamically accompany with evaluation. Some related experiments show that the recommendation method can stimulate users to improve their own trust value, and implement accurately increase of the content trust value.
\end{abstract}

\section{Introduction}

There are more and more information in the social network, which makes it difficult to find your own favorite information. So information recommendation has become the emphasis of the study of current recommendation system. The content recommendation of recommendation system is based on mutual trust. At present, study method of trust evaluation includes evaluation method of iterative satisfaction based on feedback [1], evaluation method based on fuzzy theory [2] and evaluation method based gram theory [3]. But some current evaluation model did not consider users' own attribute, just adjusting dynamically punishment or rewards function. The evaluation feedback value can be adjusted through the gram theory, and rewards or punishes real or false evaluation users. The model implements the next action in accordance with gamble result of each round, and simulates aptly users' behavior in the social network.

This paper proposes an improved recommendation method of motivating users, which calculates trust value through game theory, and motivates user evaluation. Implementing effectively content Evaluation is very important for increasing evaluation value of evaluating user. Invalid or malicious evaluation can make trust value of evaluation user down. There are some right contents or wrong contents in network information. In order to make the high accuracy rate content be evaluated, evaluation value of authority user should be introduced into the calculation of trust value. For game theory strategy, the most optimal strategy is to implement only evaluation for the right content. In 1994, S.Kamavar proposed evaluation feedback method with users trust value: EigenTrust [1]. L.Xiong proposed evaluation feedback method with users trust value: PeerTrust [4]. Yan proposed model based on trust diffusion, he believed the trust was process of dynamic spread. The model would combine content with collaborative recommendation [5]. Dong proposed the trust model based on similarity, which replaced weight of trust recommendation with similarity of users, and reduced error remarkably [6].

\section{Trust Model}

For this study, user attribute, content attribute and authority user are introduced into trust recommendation model based on the game theory. By analyzing cumulative trend of the content trust value of different attribute and trust value of evaluation user, which can motivate right evaluation and reasonable recommendation.

\subsection{Influence Factor of Evaluation.}

(1) Time. Evaluation time $\mathrm{T}=$ the current evaluation time- content post time. 
(2) User and content attribute. User attributes include some user registers information, such as major and focus area, etc. The content attribute refer to its category of content submission.

(3) User authority. User authority is formed by the user history evaluation. The user authority value can be calculated as following steps of: firstly, finding users' contribution or quantity of evaluation content, and classifying post. The weigh value $w_{i}=\left\{w_{1}, w_{2}, w_{3}\right\}, w_{i} \in[0,1]$.

$$
c=\frac{w_{1} \cdot N_{1}+w_{2} \cdot N_{2}+w_{3} \cdot N_{3}}{N_{1}+N_{2}+N_{3}}+1
$$

$N_{1}$ represents the quantity of user-generated content, $N_{2}$ represents number of the user history evaluation, $N_{3}$ represents the quantity of user' friend. The c value represents authority value of user.

\subsection{The Formal Definition of Evaluation}

Assumption 1: One user evaluates a content; The action includes evaluation and no-evaluation; The content includes right content and wrong content; The evaluation category includes evaluation and no-evaluation.

Assumption 2: More individual users evaluates a content; The evaluation category includes evaluation and no-evaluation; The benifit of each user is different at different evalutaion time; The benefit of content decreases with time delay.

Assumption 3: One user evaluate some different contents, so as to improve its own trust value; For different users, The different accuracy leads to different benifits. Users choose categeory based on their benifits.

Definition1. The basic structure of probability game model is $\langle N, S, U, P>$, where $N$ represents

users and content of game, $P$ represents the probability of users' evaluation. $P=\langle p, q>$, where $p$ represents the probability of evaluating right content, q represents the probability of evaluating wrong content. $U=<u_{a}, u_{b}>$, where $u_{a}=<u_{a 1}, u_{a 2}>$ represents the benefit of right content and wrong content, and $u_{b}$ represents users' benefit.

\subsection{The Evaluation Model}

(1)The evaluation of individual user evaluates individual content. The evaluation model analyzes the evaluation or no-evaluation of individual user. The category selection depends on the amount of benefit by using different category. The beneficial matrix is shown in Table 1.

\begin{tabular}{ccc}
\multicolumn{3}{c}{ Table.1 Pay-off matrix of exciting function } \\
\hline $\mathrm{B}$ & Right & Wrong \\
\hline Evaluation & $s 1-s 2+s 3, s 3-s 2$ & $s 3-s 2, s 3$ \\
No-evaluation & $s 1,0$ & 0,0 \\
\hline
\end{tabular}

Where $s 1$ represents the benefit that user browses right content, s2 represents consumption value that the user evaluate content, $s 3$ represents benefit of user feedback after evaluation content. $s 3=c b$,

where $\mathrm{c}$ represents authority value of the user, and $\mathrm{b}$ represents feedback value of evaluation. The abstract figures of Nash equilibrium is shown in Figrue1.

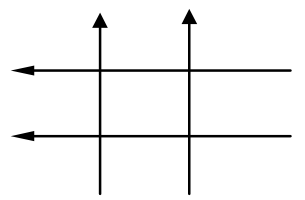

Figure.1 Nash equilibrium of exciting function

From the figure 1, the benefit of evaluation wrong content is the same as that of the right content when $c>1$. From pay-off matrix, the benefit of evaluation right content are $s 1-s 2+s 3>0$ and $s 3>s 2$. From the game, if users want to acquire higher trust value, they should exactly evaluate the right content. And content trust value depends on the value of users' authority.

(2) The evaluation of several users evaluates individual content. Because there are many users in social network, the situation of several users evaluate individual content also should be considered. 
Users' evaluations depend on their own subjective opinions. The evaluation probability of right content is $p$, and the evaluation probability of wrong content is $q$. The underappreciated probability of right content is $1-p$, and The underappreciated probability of wrong content is $1-q$. Over time, the content evaluation will be decreased, the increasing speed of trust value will get closer to 1. $\alpha$ represents the time decay function [10]. The benefit matrix of content is shown Table 2.

Table.2 Benefit of underappreciated content

$\begin{array}{cc}\text { Evaluation of rigth content } & \alpha \cdot s 3 \\ \text { No-evaluation Of rigth content } & 0 \\ \text { Evaluation of wrong content } & \alpha \cdot s 3 \\ \text { No-evaluation of wrong content } & 0\end{array}$

Assume content benefit of several users are trust value in evaluation of several users cases, the benefit of right content and wrong content have the formula (2) and formula (3) in hybrid strategies.

$$
\begin{aligned}
& u_{a 1}=p \cdot s 3 \cdot a+(1-p) \cdot 0 \\
& \frac{\partial u_{a 1}}{\partial p_{i}}=s 3 \cdot a=0
\end{aligned}
$$

Content trust value is proportional to authority value. The relationship between content trust value and authority value are shown in Figure 2.

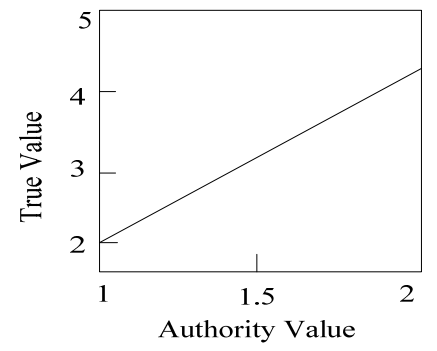

Figure.2. Change curve of trust value

From figure 2, the higher the user authority value, the higher the content trust value. So the right evaluation of authority value has a big influence on content trust value.

(3) The evaluation of individual users evaluates several contents. User can choose to evaluate several contents or not evaluate it, depending on the size of benefit. The pay-off matrix of user evaluation is shown in table 3 .

\begin{tabular}{|c|c|c|}
\hline $\mathrm{B}-\mathrm{A}$ & Right & Wrong \\
\hline Evaluation & $(s 1-s 2+s 3) a$ & $(s 3-s 2) a$ \\
\hline No-evaluation & $s 1 \cdot a$ & 0 \\
\hline
\end{tabular}

Table.3 Pay-off matrix of user evaluation

The probability of user evaluate the ith right content is $p_{i}$, and evaluate the $j$ th wrong content is $p_{j}$. we can use the equation 4 to calculate the user revenue.

$$
\begin{gathered}
u_{b}=p_{i} \cdot(s 1-s 2+s 3) \cdot a+\left(1-p_{i}\right) \cdot(s 1 \cdot a)+q_{j} \cdot(s 3-s 2) \cdot a+\left(1-q_{j}\right) \cdot 0 \\
\left\{\begin{array}{l}
\frac{\partial u_{b}}{\partial p_{i}}=(s 3-s 2) \cdot a=0 \\
\frac{\partial u_{b}}{\partial q_{j}}=(s 3-s 2) \cdot a=0
\end{array}\right.
\end{gathered}
$$

\section{Experiment and Analysis}

In order to evaluate trust value, this paper compares the model to EigenTrust model. This experiment is based on Matlab platform, and use epinion as experimental data. We choose 100 users' 
evaluation. The probability of right evaluation feedback ranges from 0 to 1 . The initial trust value is 0.5 , and other parameters are in shown in Table 4.

Table.4 Simulation parameters setting

\begin{tabular}{cc}
\hline Total number Of Evaluation & 100 \\
Probability of right evaluation & $0 \sim 100 \%$ \\
Probability of wrong evaluation & $0 \sim 100 \%$ \\
Right content & 60 \\
\hline wrong content & 40 \\
\hline User authority & $0.8,0.3$
\end{tabular}

The trust evaluation model based on game theroy has obvious growth rate, the result of growth rate is shown in Figure 3.

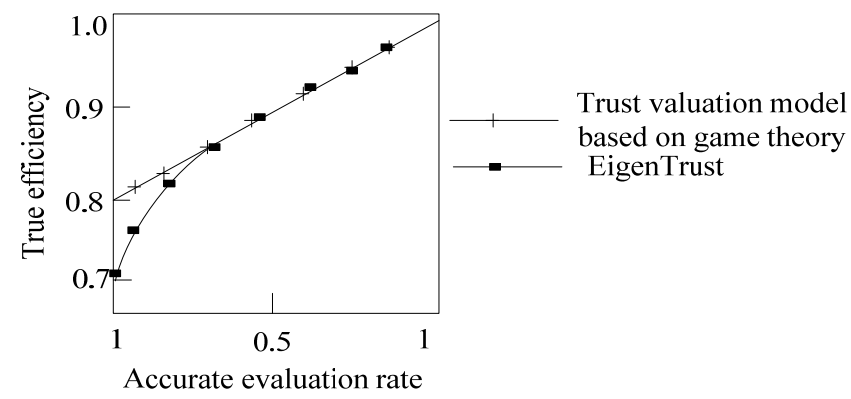

Figure.3. Effective growth rate of Trust value

As can be form the Figure 5, compared with Eigen Trust model, the effective growth rate of trust value increases as the right evaluation probability increase in improved evaluation model.

\section{Summary}

In order to solve the problem of dishonest evaluation in evaluation recommendation, this paper proposes an improved evaluation model with feedback incentive based on game theory. The model emphasizes user authority importance to evaluation. By modeling traditional evaluation model in different game stage, analyzing the benefit of user and content, the recommendation model can make user acquire the most trust in evaluation. We need introduce credibility and indirect trust values into recommendation model in the next step.

\section{References}

[1]. Kamvar. S, Sehlosser M. The Eigen Trust Algorithm for Reputation Management in P2P Networks. www, Budapest, Hungary, 2003.p.125-129.

[2]. J.LI. Web Service Trust Evaluation Model Based on Fuzzy Theory, Computer Engineering, 36(2010), No.8. p. 25-28.

[3]. L.M.SHEN, Q.SHEN,F.LI. Web Service Trust Evaluation Model Based on Game Theory, Journal of Chinese Mini-Micro Computer System, 35(2014), No.8. p. 1687-1692.

[4]. L.XIONG, L.LIU. Peer Trust: Supporting Reputation-based Trust for Peer-to-Peer Electronic Communities. Pro. Of 2004 IEEE Trans on Knowledge Data Engineering, 16(2004),No.7.p. 843-857.

[5]. J.F.YUAN. Study on Recommendation System Based on Trust Diffusion Mechanism, southwest University, China, 2008, p.36-42.

[6]. Q.WANG. Trust Evaluation Model Based on Similarity, Journal of Computer Science, 40(2013), No.16. p. 132-158. 